\title{
La práctica de la colaboración en los procesos digitales de diseño: Investigación - Acción
}

The practice of collaboration in digital processes design: Investigation action

\author{
Mario Andres Bonilla Vallejo \\ Universidad Federal de Viçosa, Brasil \\ andresvajo@gmail.com \\ Denise Mônaco dos Santos \\ Universidad Federal de Viçosa, Brasil \\ denise.monaco@ufv.br
}

\author{
Douglas Lopes de Souza \\ Universidad Federal de Viçosa, Brasil \\ dglopes@gmail.com
}

\author{
Andressa Carmo Pena Martinez \\ Universidad Federal de Viçosa, Brasil \\ andressamartinez@ufv.br
}

\begin{abstract}
This paper aims to present reflections on the practice of collaboration in the project JAM! Diálogos emergentes e processos digitais de projeto. For this, we analyzed the interaction and communication of a geographically distributed work team in Brazil, through a research - action methodology. Here be considered as main aspects the digital tools and technologies that support the development of remote architectural projects. Therefore, advances in the CSCW area taken into account for such analysis. This work linked to a master's research that be carry out at the Federal University of Viçosa
\end{abstract}

Keywords: Process design; Collaboration; Groupware; Collective intelligence.

\section{Introducción}

Los procesos remotos de diseño se caracterizan por la colaboración existente entre los equipos que están geográficamente distribuidos y trabajan concomitantes en la elaboración de un emprendimiento. Para esto, son necesarias tecnologías y herramientas que apoyen, entre los miembros del equipo, la comunicación y el intercambio de informaciones. Más aún cuando estos procesos son mediados por tecnologías digitales, las cuales permiten aumentar el número de posibles soluciones para un proyecto, creando consecuentemente, una mayor cantidad de datos.

Así pues, varios aspectos deben ser tomados en cuenta para propiciar ambientes colaborativos, como interacciones entre los miembros, espacios de trabajo, tecnologías digitales, niveles de alfabetización, entre otros.

Siendo así, este trabajo tiene como objetivo presentar reflexiones sobre la práctica de la colaboración en un equipo conformado por tres grupos de investigación: (1) NÓ.Lab Universidad Federal de Viçosa - MG; (2) Nomads Universidad de São Paulo - SP, (3) Algo+ritmo - Universidad Federal de Mato Grosso do Sul - MS, los cuales están distribuidos geográficamente en Brasil, cuyos están enfocados en la producción conjunta de conocimientos, teniendo, por tanto, el interés común de explorar la creación de una red colaborativa remota que sirva como piloto para apoyar el desarrollo de procesos de diseño a partir de la mediación digital. Estás reflexiones están basadas en la cuestión ¿Cuáles son los alcances que puede llegar a tener colaboración?
Este trabajo hace parte de un proyecto de investigación de maestría que está siendo desarrollada en base a la unión de estas tres universidades. Tal proyecto objetiva analizar la colaboración en ambientes remotos y presenciales de procesos digitales y colaborativos de diseño.

Este análisis parte de las consideraciones teóricoconceptuales presentes en la literatura académica sobre la colaboración, los procesos digitales y colaborativos de diseño y el CSCW. También está basado en un abordaje metodológico de investigación - acción, el cual, según Tripp (2005), presupone la participación del investigador implicado en los problemas investigados. La premisa de investigaciones de esta naturaleza, es que el investigador es un observador que se situá más allá del simple papel de espectador, y es, él mismo, un integrante. La observación participante, en cuanto método, se refiere al contacto directo del investigador con los actores envueltos en una acción (Tripp, 2005). En ese sentido, el investigador es, al mismo tiempo, instrumento y objeto de estudio.

Con esto en mente, este trabajo presenta un análisis participativo, en el cual se implementó el ciclo de investigación propuesto por Tripp (2005): planear, actuar, monitorear y evaluar.

Se planearon las actividades de los seminarios;

Se llevaron a cabo (actuar) los seminarios;

Se monitorearon y registraron las actividades;

- $\quad$ Se analizaron y evaluaron los datos registrados. 


\section{Trabajo [en] Conjunto}

Considerando que el ser humano es social por naturaleza, la inteligencia colectiva, intrínseca en la sociedad debe ser comprendida como un trabajo en conjunto, como punto de convergencia tanto de ideas como de personas, como una inteligencia distribuida por todas partes. De acuerdo con Lévy (2004), una inteligencia colectiva es aquella que es valorizada y coordinada en tiempo real, objetivando el reconocimiento mutuo de las experiencias y saberes de las personas.

Según Apgaua (2004), la construcción de una sociedad solidaria, colaborativa, libre, inteligente e igualitaria para todos, se da gracias a los intereses individuales que se comprenden como elementos principales para la configuración de alianzas. De esa forma, lo individual se sustituye por lo social, avanzando y solucionando problemas en conjunto, lo cual permite la satisfacción tanto de los individuos como del grupo. Así, cuanto más se desarrollan los procesos de inteligencia colectiva, los individuos y grupos se apropian con más facilidad de los cambios y, por tanto, hay menos efectos excluyentes en la sociedad (Lévy, 1997)

En ese sentido, como expresan Lévy (1997) y Apgaua (2004), en una sociedad en la que los intereses individuales se agrupan/unen y crean, por tanto, intereses colectivos, la colaboración desempeña un papel crucial para el continuo desarrollo de la inteligencia colectiva y de redes de comunicación/interacción entre diferentes agentes y culturas.

\section{Colaboración}

La colaboración puede ser pensada a partir de un contexto en el que la resolución conjunta de problemas es considerada como la base de las interacciones entre intereses individuales. Esto significa trabajar con otras personas que tienen objetivos y/o necesidades en común, intentando encontrar soluciones satisfactorias para todos los interesados (Kvan, 2000).

Ferreira (2006) define colaboración como la realización voluntaria de una tarea determinada, en la cual se satisfacen los intereses de las personas envueltas, siendo que "hay un compromiso mutuo de los participantes en un esfuerzo coordinado para la resolución conjunta del problema" (Roschelle y Teasly, apud Dillenbourg, 1996). Esto es, según Pimentell, Fuks, Lucena (2008) el hacer juntos, visando alcanzar un objetivo común.

Así pues, como lo expresan Kvan (2000), Ferreira (2006) y Pimentell, Fuks y Lucena (2008), un proceso colaborativo visa suplir los intereses y necesidades de todos, a través del trabajo en conjunto siempre que realizado de forma no jerárquica. Esto también se soporta en lo señalado por Dalkir (2011), quien afirma que el concepto representa trabajar en conjunto, en el cual los intereses individuales interactúan entre sí, generando un entendimiento común, que según el autor, "ninguno de los actores envueltos ya había poseído o no sería capaz de obtener por cuenta propia" (Dalkir, 2011).
Según John-Steiner (2000), la colaboración ofrece el establecimiento de confianza entre participantes, reuniéndolos para compartir significados y producir conocimientos al trabajar de forma complementaria.

En ese sentido, establecer colaboración es importante para crear contextos de inteligencia colectiva, en los cuales los envueltos puedan asumir riesgos, conflictos y contradicciones, debatir críticamente conceptos, valores, ideas y diferentes puntos de vista. Ya que, en ella las personas están juntas en un sentido de pertenencia, como un grupo en el que cuyos componentes interactúan entre pares con un mismo objetivo. Habiendo en él, espacio para la autonomía, igualdad, respeto mutuo y una postura de tolerancia con diferencias y divergencias (Santos, 2008)

Por lo tanto, la colaboración facilita un nuevo formato de actitudes comunitarias, que se asumen sin desvalorizarse como relaciones de beneficios mutuos, transitorias, circunstanciales e integradas al cumplimiento de metas personales sin la existencia de jerarquías.

\section{CSCW}

Computer - Supported Cooperative Work, es el nombre utilizado para designar las investigaciones en el área de trabajos realizados en conjunto, en el cual se estudia el comportamiento de los grupos al desempeñar una actividad. Para esto, se parte de las tecnologías y herramientas computacionales que pueden apoyar estos trabajos (Sarro, 2006). De esa forma, según Nitzke, Carneiro y Geller (1999), CSCW:

Trata de la cooperación en locales de trabajo, siendo la disciplina científica que describe como desarrollar aplicativos groupware, teniendo también el estudio teórico y práctico de como las personas trabajan en cooperación y como los groupware afectan el comportamiento del grupo. (Nitzke et al., 1999, p. 1)

Así, en el área de CSCW se toman como objeto de estudio las tecnologías y herramientas digitales que posibilitan el desarrollo de proyectos en grupo, considerando las diferentes formas de comunicación, cooperación y coordinación que se dan cuando actividades son realizadas en conjunto. De acuerdo con lo anterior, CSCW le ofrece a las interacciones cooperativas soporte en contextos teórico prácticos y computacionales.

Los soportes computacionales que apoyan el trabajo e interacción cooperativa se denominan groupware, y según Botelho y Vidal (2005), son definidos como "sistemas basados en computador que soportan grupos de personas comprometidas en una tarea común y que fornecen interfaces para un ambiente compartido" (Botelho y Vidal, 2005. p. 132). A su vez, estos groupware están soportados por bases de datos y servidores de redes que permiten el intercambio de recursos $y$, de esa forma, proveen las funcionalidades necesarias (Sarro, 2006). 


\section{Crowdsourcing}

El crowdsourcing es un tipo de actividad participativa en línea en la que un individuo, una institución, una organización sin fines de lucro o una empresa, proponen a un grupo de individuos de diversos conocimientos, a través de una llamada abierta flexible, el compromiso voluntario de una tarea. La realización de la tarea, de variable complejidad, en la que se debe participar aportando su trabajo, dinero, conocimiento y / o experiencia, siempre conlleva un beneficio mutuo. El usuario recibirá la satisfacción de un determinado tipo de necesidad, ya sea económico, reconocimiento social, autoestima 0 el desarrollo de habilidades individuales. (Estellés y Gonzáles, 2012, p. 9)

Este tipo de actividades están soportadas por groupware. Ejemplos de estos son Github y Trello, los cuales funcionan como repositorios basados en un sistema de control de versiones (Dabbish, et al., 2012). Ellos permiten también realizar actividades de comunicación/interacción, tales como creación de chat, foros de discusiones e incluso seguir desarrolladores altamente activos.

\section{Proceso de diseño}

Los procesos de diseño pueden estar/ser configurados y desarrollados de diversas formas. Es decir, su configuración puede estar estructurada por etapas totalmente determinadas, como lo expresa Kowaltowski (2012). Según la autora, es posible considerar los procesos de diseño como un número de actividades intelectuales básicas, las cuales están organizadas por fases: análisis, síntesis, previsión, evaluación, decisión y finalmente comunicación. Esto cuando pensadas en un orden lógico y cronológico. Sin embargo, en la práctica, algunas de ellas se pueden llevar a cabo a través de la intuición y de manera no cronológica. Ya que, de acuerdo con Terzidis (2006), los procesos son interactivos, basados en "ensayo y error", en el cual la experiencia e intuición son la base del proceso conceptivo, siendo que, su desarrollo puede estar mediado por técnicas tradicionales o técnicas digitales.

Garcia (2014), afirma que el proceso digital de diseño logra ser más directo y complejo, debido a la facilidad con que las informaciones pueden ser extraídas, intercambiadas y utilizadas, lo cual promueve un cambio en la relación que hay entre el arquitecto y las etapas de diseño. Según el autor, las herramientas computacionales soportan avanzados tipos de generación de forma, lo cual influye directamente en el objeto arquitectónico. En ese sentido, Oxman (2006), señala que aquello que era un proceso cognitivo implícito en el diseño, se tornó explicito debido a las posibilidades de interacción del diseñador con dicho objeto, de manera que, los procesos digitales de diseño no solo aumentan la complejidad del diseño, sino también la complejidad del proyecto como un todo.

En ese contexto, la colaboración surge como una aliada de los procesos de diseño, aún más cuando los miembros del equipo están distribuidos geográficamente. Según Kleinsmann (2006), la colaboración en los procesos de diseño permite que los actores de diferentes disciplinas compartan sus conocimientos con el objetivo de crear un entendimiento compartido $y$, de esa forma, explorarlo e integrarlo para conseguir el objetivo común. Sin embargo, este tipo de colaboración requiere de más exigencias, ya que, de acuerdo con Kvan (2000), el sentido de trabajar juntos es más amplio. Aquí es más difícil establecer y mantener las interacciones, el intercambio de experiencias y las responsabilidades, debido a que, para la construcción de relaciones de interacción y confianza en ambientes colaborativos, es preciso un tiempo mayor de convivencia entre los envueltos.

En trabajos anteriores sobre procesos de diseño colaborativo en instancias remotas y presenciales, se observó que sus estudios estuvieron enfocados en aspectos puntuales que posibilitaran la colaboración.

Coelho y Noaves (2008) esquematizaron el flujo de trabajo del evento BIMStorm Los Angeles, el cual tuvo como objetivo diseñar 420 edificios en 24 horas, este se enfocó en la interoperabilidad. Caballero y Hernández (2010) estudiaron el desarrollo de aplicaciones para soportar la comunicación y presentación de proyectos en entornos académicos, ofreciendo ambientes virtuales, gestión de documentos, interfaz orientada a la creación de contexto, herramientas de anotaciones, entre otras. Soetanto et al. (2015) estudio las diferencias entre los miembros de un equipo multidisciplinar respecto a sus percepciones de colaboración y formas de comunicación preferidas, tomando como base el proceso de diseño realizado por dos universidades distribuidas geográficamente. Hayes y Saleeb (2016) realizaron un comparativo entre reuniones en ambientes virtuales y ambientes presenciales, tomando en cuenta los niveles de conocimientos sobre herramientas digitales y experiencia con resolución de problemas. Boy et al. (2016) analizó la implementación de la aplicación SCORE en un equipo de diseño multidisciplinario. La aplicación visa coordinar las tareas mientras fornece a los miembros con informaciones del estado del proyecto, lo cual resulta en una visión general del mismo.

Tomando lo anterior, podemos ver que las perspectivas y enfoques de las investigaciones sobre la colaboración en los procesos de diseño, están basados en herramientas que promuevan y mejoren determinados aspectos de la colaboración, tales como interoperabilidad, comunicación, flujos de información, TIC (Tecnologías de Información y Comunicación) y gerenciamiento de proyectos, los cuales, por sí solos, no presuponen ambientes colaborativos. No obstante, juntos pueden llegar a propiciarlos.

Ahora bien, la importancia de este trabajo radica en la exploración de los alcances de la colaboración cuando pensada en un sentido más amplio. Esto es, la búsqueda de posibilidades en un mismo proceso de diseño, a través de redes de conocimiento.

\section{JAM!}

JAM! es un proyecto colaborativo que está siendo desarrollado por tres grupos de investigación de tres 
Universidades Federales de Brasil y que están distribuidas geográficamente: (1) NÓ.Lab - Universidad Federal de Viçosa - MG; (2) Nomads - Universidad de São Paulo - SP, (3) Algo+ritmo - Universidad Federal de Mato Grosso do Sul - MS. El proyecto fue configurado para que su desarrollo transcurriese de tal forma en que estuviese basado en las investigaciones que están siendo llevadas a cabo de manera individual por cada grupo. Ya que, en ellas se presentan diferentes niveles, graduación (iniciación científica), maestría y doctorado.

Cabe resaltar que cada uno de los grupos cuenta con características que los diferencian entre ellos en varios ámbitos. Uno de ellos es considerado como un grupo de investigación ya bastante consolidado, en vista de que, cuenta con un amplio número de pesquisidores en diferentes niveles y áreas de estudio, además de poseer más equipamientos y una mejor infraestructura. Mientras que los otros dos, son grupos recientes que están en fase de formación y/o consolidación, mejorando su infraestructura y adquiriendo nuevos equipos. En ese sentido, existe un desfase entre los niveles de experiencia de cada grupo

En ese orden de ideas, el equipo del proyecto JAM! está conformado por un conjunto de profesores y alumnos de Arquitectura y Urbanismo tanto de graduación como de pos graduación, con distintos niveles de experiencia y en el cual cada pesquisidor contribuye desde la perspectiva de su respectiva investigación.

El proyecto está dividido en 4 fases, las cuales tienen una duración estimada de un semestre académico: la primera fase trataba de la preparación de la infraestructura física y material para el desarrollo del proyecto, estructuración y capacitación de los grupos locales, y sistematización de los referenciales teóricos y metodológicos; la segunda fase, en curso, se proponía la elaboración remota de un proyecto por el equipo conformado por los tres grupos de investigación; la tercera fase dice respecto al desarrollo local de ese mismo proyecto por cada grupo de investigación; la cuarta y última fase trata de la producción y montaje del objeto arquitectónico en el medio urbano, y de acciones para la evaluación del proyecto.

Por tanto, en el ámbito de este proyecto, la cooperación académica entre investigadores de la UFV (Universidad Federal de Viçosa) y de las otras dos instituciones, se justifica, por un lado, por el interés común de explorar posibilidades de proyecto en red entre regiones distantes de Brasil. Por otro lado, se pretende entender, a través del uso de plataformas de comunicación y programas computacionales paramétricos y algorítmicos, como se pude dar la conexión entre el amplio ámbito de la colaboración vía web.

El proyecto comenzó como una iniciativa de estudiar procesos digitales de diseño de edificios de formas complejas, tanto en ambientes remotos como en ambientes presenciales, envolviendo concepción, fabricación y montaje. En él se implementaron procesos colaborativos de diseño, haciendo uso de plataformas, herramientas y tecnologías digitales disponibles en la internet. En lo que dice respecto a la comunicación, los tres grupos acordaron utilizar Facebook como plataforma de comunicación síncrona y asíncrona. También acordaron realizar un seminario semestral por video conferencias para exponer los avances por parte de cada grupo de investigación.

Esto a partir de las experiencias vivenciadas en la disciplina piloto que se llevó a cabo por las mismas tres universidades en el segundo semestre de 2015, denominada Arquitetura, Design Paramétrico e Fabricação Digital. Según Santos, Matrinez, Souza y Brinati (2016), "tal disciplina fue estructurada como una práctica de diseño remoto colaborativo (...) buscaba favorecer las especificidades de cada universidad y el intercambio de conocimientos a partir de experiencias locales, considerando, inclusive, las diferentes máquinas de prototipado disponibles en cada institución" (p. 131). En ella, de igual manera, se priorizó el diseño esencialmente colaborativo, puesto que, los alumnos que conformaron el grupo de esta se encontraban geográficamente distribuidos.

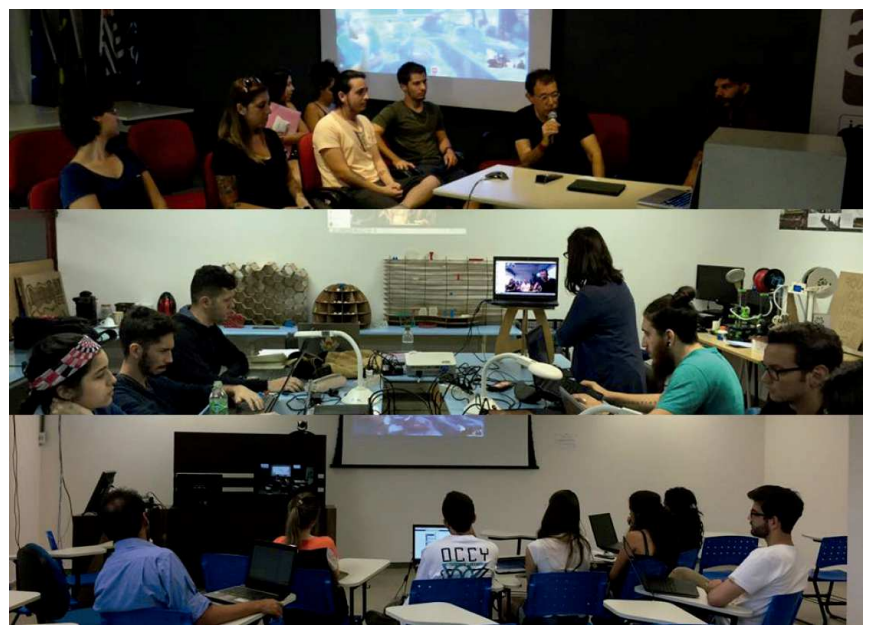

Figura 1: Encuentro semanal - Nomads.usp - Algo+Ritmo.UFMS NÓ.Lab.UFV. 2015

En el caso específico de este trabajo, se presenta el análisis de las actividades realizadas hasta la segunda fase del proyecto JAM!, exponiendo progresos, cambios y encaminamientos del mismo. Todo esto a partir de la perspectiva del grupos de investigación NÓ.Lab - UFV (Universidad Federal de Viçosa) y del respectivo Seminario II, realizado a mediados del primer semestre de 2017.

\section{Seminario II - JAM!}

El seminario conto con un cronograma desarrollado conjuntamente en tres etapas, en las cuales cada uno de los grupos tuvo espacio para exponer sus ideas: (1) trata sobre la presentación de los avances en las investigaciones que se están adelantando en cada uno de los grupos; (2) se refiere a las proposiciones sobre el proyecto, basadas en los intereses individuales de cada localidad; (3) trata de las conclusiones y nuevos rumbos que tomará el proyecto. El orden de las exposiciones fue, Algo+Ritmo, NÓ.Lab e 
Nomads, a excepción de la última etapa, la cual se dio de una forma más fluida, como una discusión abierta, de forma horizontal y sin jerarquías.

Aunque debidamente programada, la falta de coordinación entre los técnicos responsables por las salas de video conferencias de las tres universidades, sumado al uso de herramientas y plataformas no adecuadas para el intercambio de informaciones, principalmente aquellas que servirían para las presentaciones de los grupos locales, el seminario avanzo, en algunos momentos, con algunas restricciones de trocas de datos e informaciones que darían un mejor soporte a las discusiones.

Entretanto, es de resaltar que, aunque se haya optado por el uso de equipamientos exclusivos para video conferencia con el objetivo de garantizar mayor calidad en la comunicación durante el seminario, el acceso a internet desde los dispositivos móviles individuales surgió como una alternativa para el intercambio de archivos.

\section{Etapa 1 - presentación de avances en cada grupo}

Algo+Ritmo: para la presentación de avances, el grupo de investigación de la Universidad Federa de Mato Grosso do Sul preparo un video, en el cual explicaba de forma rápida los estudios que se están llevando a cabo en tal universidad. No obstante, no fue posible compartir el archivo multimedia con las otras dos universidades por medio de los equipamientos de video conferencia. Esto obligó al grupo en cuestión a proyectar de forma improvisada el video, y algunos miembros del equipo debieron recurrir a sus dispositivos móviles para lograr acceder a él, lo cual resulto en un pequeño comprometimiento en el entendimiento por parte de los otros dos grupos.

A pesar de todo, se comprendió que, están analizando la posibilidad de desarrollar parklets en asociación con órganos gubernamentales, teniendo así una docencia participativa; existen estudios de conectores de bambu para soportes fotovoltaicos; adelantan estudios en el ámbito del diseño de viviendas de emergencia junto con la secretaria de habitación, esto debido a cuestiones climáticas de la localidad; en el contexto urbano, está en desarrollo el proyecto Trocas Urbanas, en el cual se intervienen las plazas a partir de cuatro ejes principales: interactividad (realidad virtual, video sónica y grafiti digital), diálogos (conversas con arquitectos externos para discutir arquitectura), experimentos (fabricación en contextos de prototipos y de edificaciones en escala 1:1) y contexto cultural (acciones culturales, presentaciones artística con participaciones del grupo teatral "maracangalha", cine arq y video maping); también realizaron un Workshop de realidad virtual, en el cual se desarrollaron técnicas con el plugin monolit del programa Rhino, que permiten colocar algunos contextos y después combinarlos para generar parcialidades.

NÓ.Lab: En el ámbito de la Universidad Federal de Viçosa, se adelantan estudios sobre la resistencia de cuerpos de prueba producidos en impresora 3D, esto en asociación con el Departamento de Ingeniería Civil; se creó una asociación con el colectivo Formigas para que este actúe como puente entre el grupo de investigación y la comunidad del barrio Laranjal, lugar en donde se espera implementar el prototipo a escala 1:1; se integraron nuevos estudiantes al grupo, tanto de graduación como de pos graduación; está en andamiento un proyecto de ampliación/reforma del departamento de arquitectura para la construcción de un Fab - Lab, también, en ese contexto, se realizó la compra de una CNC y una cortadora laser, con el objetivo de avanzar con los estudios sobre fabricación digital.

Nomads: Destacó las investigaciones en andamiento que pueden estar relacionados con el proyecto JAM!

Estudios en BIM a partir de dos consideraciones: (1) teoría de la conversación, pensando en cómo los diversos actores envueltos en intervenciones urbanas y en especial la comunidad o representantes, pueden utilizar las plataformas; (2) procesos de diseño colaborativo a través de BIM. Están en andamiento 2 investigaciones en procesos computacionales en el uso de datos, esto de una forma más relacionada con la programación, abriendo la posibilidad de potencializar los procesos y los proyectos de diseño.

Intervenciones urbanas a través del proyecto Catografias, el cual tiene como objetivo pensar y experimentar metodologías de intervención en la ciudad. Esto a partir de medios que se cruzan y áreas que se conectan, dando así más atención al uso de los aplicativos para la manifestación directa de la comunidad. En ese sentido, también han trabajado con distintas interfaces, como las sonoras, por ejemplo, utilizadas en intervenciones de corta o larga duración, haciendo reflexionar sobre el papel del arquitecto en el espacio público. El grupo también estudia prototipado y fabricación digital en procesos digitales de diseño.

\section{Etapa 2 - Proposiciones}

Algo+Ritmo: Sugirió relacionar el proyecto JAM! con los proyectos de diseño y construcción parklets, de viviendas de emergencia, de conectores e incluso con el proyecto de Trocas Urbanas.

NÓ.Lab: Trabajar con el colectivo Formigas. La propuesta de esta unión, se dio gracias a que este colectivo se desarrolla como un proyecto de extensión ligado a la Universidad Federal de Viçosa, trabajando directamente en el barrio Laranjal. Esto con el objetivo de realizar acciones de aproximación para con la comunidad. Sin embargo, el colectivo carece de metodologías adecuadas para llevar a cabo esas aproximaciones. De esa forma, esta unión encontró varias posibilidades de entrada, una de esas fue una metodología basada en el programa spacesyntax, lo cual permite estudiar tanto la comunidad como el espacio de una forma digitalmente sistematizada. Por tanto, se pretende trabajar principalmente con spacesyntax, grasshopper y el scaner 3D, tomando los datos sociales recolectados en los levantamientos para así lograr percibir demandas reales de la comunidad. 
Por otra parte, se propuso vincular el proyecto JAM! a las investigaciones sobre la resistencia de conectores para estructuras de bambu.

Nomads: Propuso herramientas y plataformas digitales que faciliten la comunicación e intercambio de datos. Una de esas es la plataforma Github, la cual funciona como un repositorio basado en un sistema de control de versiones (Dabbish, et al. 2012). Otra cuestión propuesta por el grupo, fue utilizar estrategias de Big data, las cuales según pesquisidores el grupo Nomads, podría ser utilizada para aprovechar las informaciones sociales como entradas (input) del proyecto, así como en el caso del proyecto Cartografias Urbanas, que busca enriquecer los procesos de diseño a través de levantamientos de datos en redes sociales. Lo anterior supondría alteraciones en los procesos y proyectos de diseño como un todo.

\section{Etapa 3 - Síntesis y nuevos rumbos}

En la etapa tres del seminario, se decidió, entre otros, vincular el proyecto JAM! y el proyecto Cartografias Urbanas del grupo Nomads, ya que, ambos buscan explorar una interacción más directa con las comunidades locales. Esto a partir de la utilización de datos de entrada (input) de cuño social o socio espacial, ya sea a partir de interfaces tangibles implementadas en el medio urbano o aún más, transformándolos en parámetros de diseño. También en el ámbito del modelamiento paramétrico, y más específicamente el prototipado, se decidió ampliar las exploraciones del proyecto de conectores iniciada por el grupo Algo+Ritmo, estableciendo, implícitamente, una asociación con las experiencias desarrolladas con los cuerpos de prueba por parte del grupo NÓ.Lab y el Departamento de Ingeniería Civil de la Universidad Federal de Viçosa.

En ese sentido, se percibió, en términos generales y más allá de lo descrito anteriormente, por un lado, hay inúmeros vínculos entre las investigaciones en andamiento de los grupos, y por otro, las posibilidades de trocas específicas de informaciones y conocimientos podrían enriquecer otros procesos que parecen ser más amplios que aquel primer objetivo de desarrollar un único proyecto por el equipo compuesto por los tres grupos de investigación. De esa forma, las posibilidades de colaboración entre grupos distribuidos geográficamente parecen estar delineadas a partir de nuevos procesos y formas específicas de diseño, para lo cual se consideró que la comunicación asíncrona sería más apropiada a ese contexto. Por tanto, esto presupone una forma diferente de trabajar simultáneamente. Aquí se habla de tiempos asíncronos para que los conocimientos se amplíen, como sugirió el grupo Nomads.usp. Para esto, se indicó emplear, de forma crítica, plataformas colaborativas como Github, Trello y otras, analizando, a su vez, posibilidades y limitaciones de las mismas.

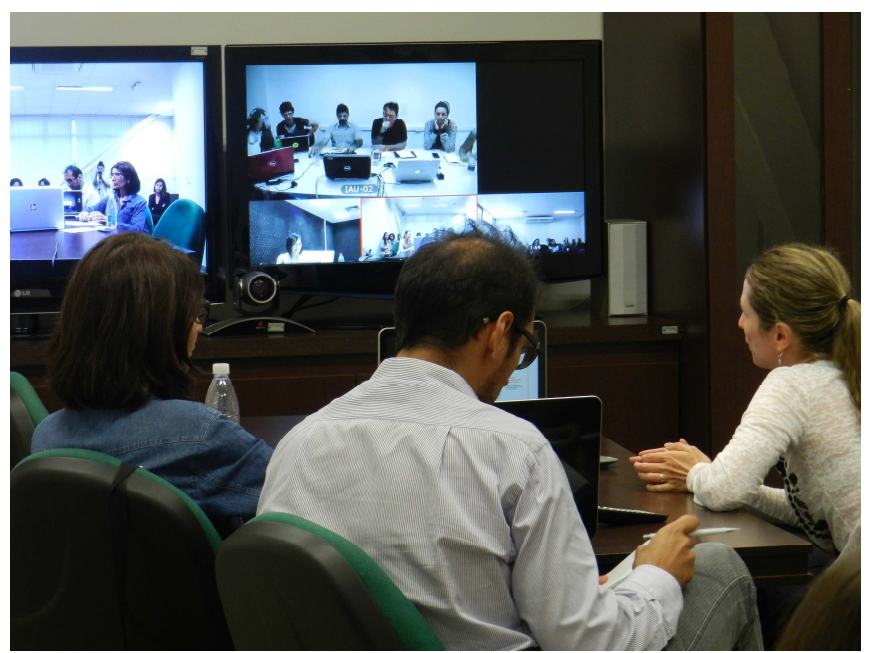

Figura 2: Seminario II del proyecto JAM!

\section{Discusión}

A partir de este seminario se establecieron más que nuevas tareas, se delinearon nuevos rumbos para el desarrollo de las siguientes fases del proyecto JAM!, inclusive para la propia fase dos, que está en curso.

Del desarrollo conjunto de un proyecto que pudiese desdoblarse en tres proyectos con determinantes locales, se pasó a privilegiar la exploración conjunta de procesos digitales de diseño, partiendo de un abordaje más metodológico. Así, se asume incluir las diferentes perspectivas de desarrollo que un proyecto de arquitectura trae consigo cuando envuelve medios digitales. Siendo así, se prioriza el intercambio de informaciones y conocimientos a partir de la consideración y manutención de los diferentes enfoques de cada investigación. Por consiguiente, se consideran las particularidades y contextos propios, en los que cada grupo y localidad se encuentran envueltos, y es, de esa forma, que el proyecto JAM! reafirma la colaboración y la creación de una "inteligencia colectiva" a partir de los intereses individuales de cada grupo de investigación, puesto que, se busca un equilibrio entre las aptitudes, habilidades, o todavía, las necesidades particulares y el conjunto común de intereses, considerando que el intercambio de informaciones pueda ser ampliado a la búsqueda de soluciones y nuevas formas de diseño. . Esto es, una red colaborativa en la que los conocimientos están disponibles para todo aquel que los necesite.

Como presentado arriba, se percibe que el diseño digital en arquitectura se da a partir de diferentes dimensiones, las cuales van desde aspectos de intervenciones en contextos urbanos y comunitarios basados en distintas interfaces, hasta aspectos de fabricación digital de conectores.

Aunque el proyecto JAM! está finalizando la fase dos de cuatro, es posible inferir divergencias entre su proceso y el proceso de la disciplina Arquitetura, Design Paramétrico e Fabricação Digital. 
A pesar de que en la disciplina se tomaron en cuenta los desfases existentes respecto a las habilidades $y$ conocimientos por parte de los alumnos, no se esperaba la jerarquización presentada en algunas etapas del proceso, lo que consecuentemente afecto las discusiones, tornándolas, por tanto, menos participativas. Esto sumado a la (in)disponibilidad de tiempo en común con la que contaban. Por otra parte, ya que el objetivo del JAM! se alteró y/o amplio para contextos colaborativos en los cuales se prioriza la interacción entre los miembros del equipo, las habilidades particulares y el aumento de los conocimiento de forma conjunta, se suprime, de cierta manera, el surgimiento de formas jerárquicas. Aquí, el intercambio de ideas se da gracias a las relaciones personales, en las cuales los individuos con diferentes aptitudes se unen en torno de una problemática, y de esa forma, este proceso potencializa el surgimiento de nuevas ideas. Esto a partir de la interacción e intersección de áreas de conocimiento similares y complementarias sobre el objeto de estudio.

\section{Conclusiones}

El proyecto JAM! puede comprenderse como un crowdsourcing, ya que, este parte de una llamada abierta de colaboración para el desarrollo de una tarea específica, en la cual se hacen necesarios diversos tipos de conocimientos, experiencias y equipos.

Así lo entiende el Ministerio de Ciencia, Tecnología e Innovación de Brasil, el cual en su propuesta de ESTRATÉGIA NACIONAL DE CIÊNCIA, TECNOLOGIA E INOVAÇÃO 2016 - 2019 (2016) considera diferentes escalas espaciales (local, regional, nacional y global) para las inversiones en investigación, desarrollo e innovación (PD\&l), ya que, objetiva la formación de redes colaborativas de investigación. Las redes de colaboración fornecen las universidades con el acceso a informaciones, recursos, nuevas áreas de conocimiento e incluso tecnologías. En este contexto, es posible que se presente una economía de alcance gracias a redes colaborativas, puesto que, ocurre una reducción de costos de producción y refinamiento del producto cuando un mismo producto puede ser producido por/para dos o más investigaciones. Siendo que, la participación en red permite que los grupos intercambien recursos necesarios para desarrollar sus investigaciones y tecnologías, lo cual extrapola los límites tanto institucionales como nacionales. Un ejemplo de esto es el proyecto JAM! En él, los recursos que son invertidos en una de las tres universidades son compartidos con las otras dos. Esto es, tres locales diferentes con distintos conocimientos, experiencias y equipamientos, aportando diferentes resultados y puntos de vista para la misma investigación. Así, la colaboración en línea permite realizar inmediatamente envíos y pruebas de las hipótesis propuestas por cada grupo, al mismo tiempo que genera documentos de registro de tales informaciones, lo cual permite la exposición del proceso. Por tanto, es posible experimentar con otros equipamientos sin la necesidad de adquirirlos, es decir, la colaboración también contribuye con la optimización de recursos y tiempo.
La colaboración puede presentarse en diferentes contextos y por ende, dentro de ellos, en diferentes niveles. En el contexto académico, esta se puede presentar en los niveles interpersonales, intergrupales, interdisciplinarios, interinstitucionales, etc., acoplándose y, por consiguiente, reconfigurando sus características.

En síntesis, la colaboración lleva consigo, implícitamente, aspectos y niveles que precisan ser explorados más a fondo, para así provechar todas sus potencialidades, no solo como metodología de trabajo, sino también como práctica continua en las relaciones sociales.

\section{Agradecimientos}

A la FAPEMIG - Fundação de Amparo à Pesquisa de Minas Gerais, por el apoyo al proyecto "JAM! Diálogos Emergentes e Processos Digitais de Projeto" (proceso TEC-APQ-0092415) al cual esta investigación está vinculada.

\section{Referencias}

Apgaua, R. (2004, Ene). O Linux e a perspectiva da dádiva. Horizontes antropológicos, (21), p.221-224.

Botelho, E. \& Vidal, J. (2005, May). Trabalho cooperativo suportado por computador. Holos, (21), p. 130-137.

Boy, G., Jani, G., Manera, A., Memmott, M., Petrovic, B., Rayad, Y. et al. (2016). Improving collaborative work and project management in a nuclear power plant design team: A humancentered design approach. Annals Of Nuclear Energy, 94, 555565. http://dx.doi.org/10.1016/j.anucene.2015.12.039

Coelho, S. B; \& Novaes, C. C. (2008) Modelagem de Informações para Construção (BIM) e ambientes colaborativos para gestão de projetos na construção civil. VIII Workshop Brasileiro - Gestão do Processo de Projetos na Construção de Edifícios 2008. São Paulo, Brasil.

Caballero, H., Hernández, J. (2010, Nov 17 - 19). Un ambiente de telecolaboración para el análisis de proyectos en arquitectura. SIGraDi 2010, XIV Congreso de la Sociedad Ibero-americana de Gráfica Digital. Bogotá, Colombia.

Dabbish, L., Stuart, C., Tsay, J. \& Herbsleb, J. (2012, Feb 11 - 15). Social Coding in GitHub: Transparency and Collaboration in an Open Software Repository. CSCW'12, Washington D.C.

Dalkir, K. (2011) Knowledge Management in Theory and Practice. Oxford, Inglaterra: Elsevier.

Dillenbourg, P., Baker, M., Blaye, A. \& O'Malley, C. (1996). The evolution of research on collaborative learning. En: E. Spada, P. Reiman. (Ed.), Learning in Humans and Machine: Towards an interdisciplinary learning science (p.189-211). Oxford, Inglaterra: Elsevier.

Estellés, E., \& Gonzáles, F. (2012). Towards an integrated crowdsourcing definition. Journal of Information Science. (38), p189-200.

Ferreira, M. (2006, Feb). Requisitos e arquitetura para sistemas de apoio à colaboração nas fases iniciais do processo de projeto. Tesis de Doctorado. Universidad Federal de Santa Catarina. Florianópolis, Brasil.

Garcia, D. (2014). Arquitetura performativa: a utilização do dprofiler para elaboração da forma arquitetônica. Disertación de maestría. Faculdade de Arquitetura e Urbanismo. Universidade de Brasília. Brasília, Brasil. 
Hayes, E., \& Saleeb, N. (2016 May. 12 -14). The virtual interactive relationship between bim project teams. Congress of Architectural Technology 2016, Conference Proceedings of the 6th International. University of Alicante. España.

John-Steiner, V. (2000). Creative Collaboration. New York, EEUU: Oxford University Press.

Kowaltowski, D.; Moreira, D. C. \& Deliberador, M. S. (2012). O programa arquitetônico no processo de projeto: discutindo a arquitetura escolar, respeitando o olhar do usuário. En: M. Salgado; P. Rheingantz. (Ed), Projetos Complexos e os Impactos na Cidade e na Paisagem (p. 85-102). Rio de Janeiro: Editora da UFRJ.

Kvan, T. (2000, Jul). Collaborative design: what is it? Automation in construction. (9), p. 409-415.

Kleinsmann, M. (2006). Understanding Collaborative Design. Wateringen, Netherlands: JB\&A grafische communicatie.

Lévy, P. (2004). Inteligencia colectiva: por una antropología del ciberespacio. Washington, D.C., EEUU: Organización Americana de la salud.

Lévy, P. (1997). Cyberculture. Rapport au Conseil de l'Europe dans le cadre du projet. Francia: Ed. O. Jacob.

Ministério da Ciência Tecnologia e Inovação (MCTI). (2016) Estratégia Nacional de Ciência, Tecnologia e Inovação 20162019. Brasília, Brasil. Disponible en http://www.mcti.gov.br/documents/10179/1712401/Estrat\%C3\%A 9gia+Nacional+de+Ci\%C3\%AAncia\%2C\%20Tecnologia+e+Inov a\%C3\%A7\%C3\%A3o+2016-2019/0cfb61e1-1b84-4323-b136$8 \mathrm{c} 3 \mathrm{a} 5 \mathrm{f} 2 \mathrm{a} 4 \mathrm{bb} 7$

Oxman, R. (2006). Theory and design in the first digital age. Haifa: Faculty of Architecture and Town Planning Technion.

Pimentel, M., Fuks, H. \& Lucena, C.J.P. (2008). Um Processo de Desenvolvimento de Sistemas Colaborativos baseado no Modelo 3C: RUP- 3C-Groupware", Anais IV Simpósio Brasileiro de Sistemas de Informação. Rio de Janeiro: UNIRIO. Disponible en http://www.les.inf.puc-rio.br/groupware

Santarosa, L., Nitzke, J., Carneiro, M. \& Geller, M. (1999, Nov) Criação de ambientes de aprendizagem colaborativa. X SBIE. Curitiba.

Santos, D. (2008). Espaços híbridos na cidade: interfaces computacionais para comunidades locais. Tesis de doctorado. Escola de Engenharia de São Carlos. Universidade de São Paulo. São Carlos, Brasil.

Santos, D., Matrinez, A., Souza, D. \& Brinati, P. (2016, Nov 9 - 11). Interfaces em Ação: sobre interações e layers comunicacionais nas práticas de projeto remote. SIGraDi 2016, XX Congreso de la Sociedad Ibero-americana de Gráfica Digital. Buenos Aires, Argentina.

Sarro, C. (2006). Implementação de uma política de colaboração para um ambiente de co-autoria de diagrama de classes. Disertación de Maestría. Universidad Metodista de Piracicaba. Piracicaba, Brasil.

Soetanto, R., Childs, M., Poh, P., Austin, S., Glass, J., \& Adamu, Z. et al. (2015). KEY SUCCESS FACTORS AND GUIDANCE FOR INTERNATIONAL COLLABORATIVE DESIGN PROJECTS. International Journal Of Architectural Research, 9(3), 6 - 25.

Terzidis, K. (2006). Algorithmic architecture. Burlington, USA: Architectural Press \& Elsevier.

Tripp, D. (2005, Sep-Dic) Pesquisa-ação: uma introdução metodológica. Educação e Pesquisa. (31), p. 443-466. 